\title{
化学实验教学中加强诚信教育的探索与实践
}

高玉霞, 聂海瑜, 路慧哲, 张晨辉, 杜凤沛 ${ }^{*}$

中国农业大学理学院, 北京 100193

摘要: 诚信是立德树人的重要内容, 关系到大学生的成才与社会发展。化学实验课程的特点有利于诚信教育的开展, 为 了充分了解大学生的诚信意识及实验教学中是否存在诚信缺失等问题, 我们开展了相关的问卷调查。综合调查结果 及教学过程中的观察, 总结出目前实验教学中存在的失信问题, 并针对这些问题在教学中进行了诚信教育实践。

关键词: 化学实验; 实验教学; 诚信教育; 学术规范; 问卷调查

中图分类号: G64; O6

\section{Exploration and Practice of Strengthening Integrity Education in Chemistry Laboratory Teaching}

\author{
Yuxia Gao, Haiyu Nie, Huizhe Lu, Chenhui Zhang, Fengpei Du * \\ College of Science, China Agricultural University, Beijing 100193, China.
}

\begin{abstract}
Integrity is an important part of moral education, which is related to the growth of college students and the development of society. The characteristics of chemistry laboratory class are conducive to the implementation of integrity education. For better understanding the integrity awareness of college students and the academic dishonesty in current laboratory teaching, we conducted relevant survey using questionnaires. Combined with the investigation results and the observation from teachers in the teaching process, the dishonest problems in the current laboratory teaching are summarized, then the related practice of integrity education was carried out in the laboratory teaching.
\end{abstract}

Key Words: Chemistry experiment; Laboratory teaching; Integrity education; Academic norms; Questionnaire

习近平总书记在全国高校思想政治工作会议上强调: “要坚持把立德树人作为中心环节, 把思想 政治工作贯穿教育教学全过程, 实现全程育人、全方位育人, 努力开创我国高等教育事业发展新局 面 ${ }^{[1]}$ 。由此可见, 教育的根本任务是立德树人。诚信教育作为立德树人的重要内容, 直接关系到大学 生的健康成长, 是大学生顺利成长成才的根基, 也是社会发展的需要 ${ }^{[2]}$ 。同时, 大学生作为未来国家 建设的主力军和科研的后备力量, 他们的诚信意识会直接关系到我国科学研究领域的学术风气及国 家的健康发展。

大学期间, 正值大学生人生观、世界观、价值观形成的重要阶段, 加强对大学生诚信教育有利 于大学生诚信价值观的建立和诚信行为方式的养成。但是就目前来看, 高校并没有针对本科生开设 专门的诚信教育课程或学术规范指导讲座等, 局限于通过思想政治课程渗透和班会主题教育开展, 采

收稿: 2020-06-29; 录用: 2020-07-23; 网络发表: 2020-09-02

“通讯作者, Email: dufp@cau.edu.cn

基金资助: 国家自然科学基金(21802166); 中国农业大学理学院教育教学改革项目 
用传统的说教模式对学生进行诚信理念的传输 ${ }^{[3-5]}$ 。这些教育途径形式相对单一、碎片化、重理论轻 实践, 大多停留在理论教育阶段, 缺乏对学生实际的德育指导和规范, 容易让学生产生抵触情绪, 导 致诚信教育的实效性和长效性不足 ${ }^{[6]}$ 。只有将诚信教学理论与诚信教学实践相结合, 将诚信意识付 诸实践, 才能达到诚信教育的目的。

化学是一门以实验为基础的学科, 许多化学理论和规律的获得都是从实验中总结出来的 ${ }^{[7]}$ 。在 化学及相关专业人才的培养过程中, 实验课是一个十分重要的实践教学环节。由于化学实验课对学 生操作的自由度相对较大, 这样的课程特点非常有利于进行诚信教育。与理论课的教学模式相比, 实 验课上教师有更充足的时间与学生面对面交流, 可以仔细观察学生的实验操作, 能够深入了解学生, 可以更有针对性地进行教育 ${ }^{[8-10]}$ 。在实验教学中加强对诚信意识的培养, 使学生在入学初期就能处 于一种严谨的科学研究氛围, 养成良好的实事求是的科研习惯和追求真理的科学态度, 对我国未来 科学研究、创新能力、人才培养、学术风气乃至社会道德都将产生积极的作用。

\section{1 目前实验教学中的诚信现状分析}

\section{1 针对学生开展的诚信问卷调查}

为了充分了解授课学生的诚信意识及目前实验教学中是否存在学术不规范等问题, 我们面向化 学、食品、生物、环境工程等专业 2017 级和 2018 级学生开展了相关的问卷调查, 发放问卷 353 份, 回收有效问卷 320 份。问卷内容主要包括学生对诚信的认识、对诚信缺失的态度、实验教学过程中 出现的问题等。期望通过这样的调查问卷形式, 能够清楚地了解我校学生的诚信意识以及目前实验 教学中学术诚信的现状, 为今后在基础实验教学中加强诚信教育提供一定的参考。

在问卷设计过程中, 为了保证调查结果的真实性和有效性, 在问卷开端指导语部分, 向学生介 绍此次调查的目的, 承诺对调查结果保密, 仅供教学参考, 以解除学生的犹豫心理, 从而真实作答。 在此基础上, 题目的设计除了考虑语言清晰、简明扼要、逻辑严谨外, 还要保证问卷的完整性和层 次性。具体来讲, 围绕着 “物理化学实验课诚信现状分析” 的主题, 问卷题目不仅涵盖学生实验课 诚信行为的所有方面, 包括实验操作、数据记录、报告书写等全部环节, 而且题目的设计和排序还 要符合调查内容的逻辑。此次问卷设计中, 将调研内容自上而下主要分为 5 个层次: 对学生整体诚 信意识的了解、教学中诚信缺失的具体表现、学生对诚信教学的态度、诚信缺失的原因、对教学的 建议, 并基于这样的层次对问题进行排序, 实现层层递进。问卷共设计 12 道相关题目, 具体题目设 计思路及调查结果分别见表 1 和表 2 。

从问卷调查的整体结果来看, 新生代大学生的诚信意识还是非常好的, 无论是个人诚信问题还 是实验教学过程中的表现。具体到细节问题上, 学生们的反馈各不相同。从问题 1 学生的自我诚信 评价可以看出, $85 \%$ 的学生认为自己非常诚信, $15 \%$ 的学生表示大多数情况下都是诚信的, 说明目前 大学生的诚信意识比较好; 从问题 2-5 的统计结果可知, $80 \%-85 \%$ 的学生在实验操作和数据处理过 程中能够做到实事求是, 而 $15 \%-20 \%$ 的学生会表现出一定的不诚信行为, 比如打碎仪器私藏起来、 私自缩减实验量、修改部分数据等; 从问题 6 和 7 可以看出, 在实验报告书写过程中, $90 \%$ 以上的 学生能够做到独立思考和书写, 仅有不到 5\%的学生可能会直接抄袭报告; 从问题 8-10 结果可知, 所有学生都认为在实验课中保持诚信很重要, 但是却有 $32.5 \%$ 的学生觉得实验课出现的修改数据或 抄袭报告算不上学术不规范, 也有超过 70\%的学生面对这些不规范行为时, 觉得与自己无关, 持冷 漠态度。这些结果非常值得我们关注, 说明学生虽然觉得诚信很重要, 但对诚信与学术不规范的理 解却不够全面。这也提醒我们在后续的实验教学中, 需要加强对学生学术规范的培养, 教导学生什 么是规范, 如何养成严谨求实的科研态度。从问题 11 可以看出, 有一半的学生认为追求更好的成绩 是当下大学生实验数据造假的主要原因, 其余学生认为缺乏学术道德教育以及教师惩罚较少也是学 术不规范的主要原因。这一结果为实验教学中加强诚信教育提供了参考, 教师可以通过改革成绩评 价方式、进行学术道德示范、加强对造假行为的处罚等措施来改善诚信缺失的现状。问题 12 的结果 
为教师对学术不规范行为的处罚措施提供了具体建议, 大部分学生觉得应该通过扣分和实验重做减 少造假行为, 少数学生觉得可以采取批评教育和口头警告来处罚。

表 1 调查问卷题目设计思路

\begin{tabular}{lll}
\hline 序号 & \multicolumn{1}{c}{ 调查主题 } & \multicolumn{1}{c}{ 涉及教学环节 } \\
\hline 问题 1 & 对学生整体诚信意识的了解 & 无 \\
问题 2-7 & 实验教学中诚信缺失的具体表现 & 实验操作、实验记录、数据处理、报告书写 \\
问题 8-10 & 学生对诚信教学的态度 & 数据处理、报告书写 \\
问题 11 & 实验教学中诚信缺失的原因 & 课程评价 \\
问题 12 & 学生对诚信教学的建议 & 无 \\
\hline
\end{tabular}

表 2 调查问卷结果统计分析

\begin{tabular}{|c|c|c|}
\hline 序号 & 问题 & 问卷结果 \\
\hline 1 & 你认为自己是讲诚信的人吗? & $\begin{array}{l}85 \% \text { 的学生认为自己是一个非常讲诚信的人, } 15 \% \text { 的学生认为自己基本 } \\
\text { 上比较诚信, 会视具体情况而定 }\end{array}$ \\
\hline 2 & $\begin{array}{l}\text { 你是否有过为了尽快完成实验而编造数据或私自 } \\
\text { 减少实验量的行为? }\end{array}$ & $82.5 \%$ 的学生表示自己从来没有, $17.5 \%$ 的学生表示偶尔有 \\
\hline 3 & $\begin{array}{l}\text { 在实验过程中, 如果不小心打碎了仪器或弄坏设 } \\
\text { 备, 你会怎么做? }\end{array}$ & $\begin{array}{l}95 \% \text { 的学生表示会主动告诉老师, 并承认错误; } 5 \% \text { 的学生表示会在不 } \\
\text { 影响自己实验前提下, 悄悄藏起来 }\end{array}$ \\
\hline 4 & $\begin{array}{l}\text { 实验课给老师检查数据时, 你的数据是否是真实 } \\
\text { 的原始数据? }\end{array}$ & $\begin{array}{l}85 \% \text { 的学生是完全由自己实际测得的真实数据, } 12.5 \% \text { 的学生会根据标 } \\
\text { 准答案优化自己的数据, } 2.5 \% \text { 的学生会参考别的同学的数据, 适当修 } \\
\text { 改自己的数据 }\end{array}$ \\
\hline 5 & $\begin{array}{l}\text { 当你处理实验数据发现自己的数据与理论值相差 } \\
\text { 很多时, 你会怎么做? }\end{array}$ & $\begin{array}{l}82.5 \% \text { 的学生会严格按照实验数据处理, 即使不能得出正确的结论, } \\
17.5 \% \text { 的学生对数据进行少量的修改, 使实验结论尽可能靠近理论值 }\end{array}$ \\
\hline 6 & 你的实验报告是否独立完成? & $\begin{array}{l}17.5 \% \text { 的学生会自己完全独立完成, } 82.5 \% \text { 的学生偶尔和同学讨论后自 } \\
\text { 己处理, 没有学生直接抄袭 }\end{array}$ \\
\hline 7 & 你身边有同学抄袭实验报告吗? & $2.5 \%$ 的学生表示比较多, $62.5 \%$ 的学生表示很少, $35 \%$ 的学生表示没有 \\
\hline 8 & $\begin{array}{l}\text { 如果你发现身边的同学有抄袭同学实验数据或编 } \\
\text { 造数据的行为时, 你会怎么做? }\end{array}$ & $\begin{array}{l}5 \% \text { 的学生会向老师反映, } 22.5 \% \text { 的学生会当面劝阻, 而 } 72.5 \% \text { 的学生不 } \\
\text { 予理会 }\end{array}$ \\
\hline 9 & 你觉得化学实验课程中是否有必要保持诚信? & $100 \%$ 的学生都认为非常有必要, 要尽早养成诚信的科研习惯 \\
\hline 10 & $\begin{array}{l}\text { 你觉得化学实验课中, 数据作假或抄袭报告是否 } \\
\text { 属于学术不规范? }\end{array}$ & $\begin{array}{l}67.5 \% \text { 的学生认为这种行为非常不好, 属于学术不规范, 应该严厉禁止, } \\
\text { 而 } 32.5 \% \text { 的学生觉得还好, 算不上学术不规范 }\end{array}$ \\
\hline 11 & $\begin{array}{l}\text { 你认为目前实验教学中存在不诚信现象的最主要 } \\
\text { 原因是什么? }\end{array}$ & $\begin{array}{l}27.5 \% \text { 的学生认为是学生缺乏学术道德和诚信教育, 态度不够端正, } \\
17.5 \% \text { 的学生认为失信成本低, 学校和老师不会惩罚, 而 } 55 \% \text { 的学生认 } \\
\text { 为是为了取得更好的成绩 }\end{array}$ \\
\hline 12 & $\begin{array}{l}\text { 如果在化学实验课中, 出现了学术不规范行为, } \\
\text { 你认为老师应该如何做? }\end{array}$ & $\begin{array}{l}37.5 \% \text { 的学生认为应该本次实验重做, } 47.5 \% \text { 的学生认为应该酷情扣分, } \\
10 \% \text { 的学生认为应该当面教育, } 5 \% \text { 的学生觉得应该进行口头警告 }\end{array}$ \\
\hline
\end{tabular}

\section{2 实验教学中出现的主要诚信问题}

结合教师在教学中的长期观察, 目前实验课教学过程中学生表现出来的失信问题依然较多。主 要有以下几种情况: (1) 为了追求标准答案和高分, 不能本着实事求是的态度去完成报告, 常出现修 改实验数据和编造实验现象的问题; (2) 在实验过程中为了尽快达到误差范围内的平行值, 人为缩 减实验量编造数据; (3) 直接抄袭别人的实验报告, 出现实验结果和实验原始记录不符; (4) 实验过 
程中, 打碎玻璃仪器、弄坏实验设备、污染公共试剂后, 并没有及时告知老师, 而是悄悄掩盖, 影 响别的同学实验的开展。这些不诚信的行为, 都让我们清楚地认识到在化学实验中加强诚信教育和 学术规范迫在眉睫。

\section{2 在实验教学中开展诚信教育的实践}

综合问卷调查的结果和上课中发现的问题, 我们在物理化学实验课程中开展了诚信教育的实践, 希望可以引导学生树立正确的学术道德观, 培养他们实事求是的科学态度。具体包括以下几个方面。

\section{1 加强教师师德建设, 发挥榜样作用}

作为实验教学的具体组织者和实施者, 教师的言行举止会对学生产生潜移默化的影响。只有教 师具备了良好的诚信意识和学术道德, 才能以身作则, 真正将这种诚信教育融入化学实验教学中, 最终才能引导学生形成严谨求实的科学态度 ${ }^{[11]}$ 。因此, 在实验教学中开展诚信教育的前提是要先加 强教师的师德建设, 树立诚信的典范, 发挥榜样作用, 真正做到陶行知先生所说的 “千教万教教人 求真”。

\section{2 加强学生诚信教育, 坚持示范性与渗透性相结合}

在实验课教学环节, 教师要坚持示范性与渗透性相结合, 利用专业知识和教学手段对学生进行 诚信意识的渗透, 以循序渐进、潜移默化的方式将诚信的理念注入学生的思想意识中。为了帮助学 生养成良好的科研习惯与学术道德, 教师通过主动示范, 告诉学生最正确的处理方式, 包括课前预 习、实验操作、实验现象的记录、数据处理、误差分析等环节, 减少学生因不清楚什么是 “规范” 而随意操作。同时, 在授课时可以将诚信素材融入其中, 包括社会上的热点新闻案例, 激发学生的 诚信意识, 教导学生在科学研究中诚实守信, 促使他们树立诚信的人生观。

\section{3 加强课前预习、课堂监督、课后讨论, 培养规范的实验习惯}

在物理化学实验课程中, 为了培养学生的规范操作, 分别加强了对学生课前预习、课堂操作、 课后讨论环节的考查。教学团队采用多媒体教学手段, 将每个实验的标准操作制作成微视频上传至 教学平台, 作为教学资源供学生下载学习。学生通过视频预习加深对相关操作的认识和对整个实验 流程的熟悉, 有利于养成规范操作的习惯。课堂中, 教师会采用抽查的方式来检查学生们的预习效 果, 让学生自己讲实验和注意事项, 其他同学补充提问, 以此增加他们对实验的理解。实验结束后, 加强对实验现象和结果的讨论, 使学生从根本上理解实验结果出现的原因, 有利于加深他们对实验 的思考和书写出更有深度的实验报告。

\section{4 优化课程评价方式, 注重过程和能力考核}

在调查学生数据造假原因中, 发现有一半学生的动机是追求更好的成绩。为了预防这些不良现 象的发生, 实验课教学要优化课程评价方式, 注重过程和能力考核, 降低实验结果在考核中所占比 例。实验课的教学目的不仅是传授专业知识, 更多地在于对学生实验操作技能、科学思维方法、严 谨求实的科学态度、创新能力、发现问题、分析问题、解决问题能力等多种科学素养的综合训练。 因此, 数据的标准与否不影响成绩, 实验过程得到的锻炼和真实的数据更重要。此外, 还要鼓励学 生积极创新、大胆思考, 对不同的实验现象和数据有自己的见解, 激发他们的科研兴趣。

\section{5 提高失信成本, 加大学术造假惩罚力度}

大学校园里失信现象屡禁不止, 大多是缺乏处罚的依据, 最终采取了宽容的态度, 没有给予处 罚。但这样的纵容反而导致部分学生继续心怀侥幸, 胆大妄为, 引起失信行为的协同和放大。因此, 为了保持科学研究的严谨和真实, 必须要提高失信成本, 加大对学术造假的惩罚力度。在实验教学 中, 一旦发现学生修改编造数据或抄袭报告, 教师要给予严厉的批评警告, 以零分处理; 对于其他 一些损坏仪器私自处理、替他人签到、私自缩减实验量等失信行为, 也要及时批评教育, 适当减分。 


\section{3 结语}

当代大学生肩负着全面建设小康社会、实现中华民族伟大复兴的使命。高等学校教育者应该努 力帮助大学生实现这一梦想, 并向国家和社会输送合格的人才。结合化学实验课的教学目标和教学 特点, 将诚信教育融入其中, 在培养学生科学素养和创新能力的同时, 帮助学生建立诚信道德的人 生观, 并将其付诸实践。经过前期的反复探索与实践, 我们也必须认识到, 在化学实验教学中开展 诚信教育是一项长期性和系统性的工程, 需要循序渐进和逐步优化培养方式, 从而更好地提高大学 生的科学素养和人文道德, 全面实现人才培养的目标, 最终推动社会和谐发展。

\section{参 考 文 献}

[1] 习近平在全国高校思想政治工作会议上强调: 把思想政治工作贯穿教育教学全过程 开创我国高等教育事业发展新局面. 中国共产党新闻. [2020-06-20]. http://dangjian.people.com.cn/n1/2016/1209/c117092-28936962.html

[2] 宋猛. 教书育人(高教论坛), 2020, $12,10$.

[3] 于丽. 思想政治教育研究, 2018, 34 (2), 22.

[4] 黄瑞. 西部素质教育, 2020, 6 (8), 33.

[5] 徐海燕. 广西青年干部学院学报, 2019, 29 (3), 23.

[6] 唐吴. 当代教育实践与教学研究, 2020, 5, 227.

[7] 王自军. 广东化工, 2009, 36 (2), 105.

[8] 薛斌. 大学化学, 2018, 33 (12), 28.

[9] 范森, 朱元海. 大学化学, 2017, 32 (7), 72.

[10] 王永红, 周先波, 魏旻辉, 毛红雷. 广东化工, 2017, 44 (17), 209.

[11] 李明菲, 何志平. 学校党建与思想教育, 2020, 9, 74 . 